\title{
Application of Chaos Theory in Identification of Two-Phase Flow Patterns and Transitions in a Small, Horizontal, Rectangular Channel
}

\author{
Y. Cai, M. W. Wambsganss, and J. A. Jendrzejczyk \\ Energy Technology Division \\ Argonne National Laboratory \\ Argonne, Illinois 60439
}

This work was supported by the U.S. Department of Energy, Office of Conservation and Renewable Energy, Division of Advanced Industrial Concepts, and represents a U.S. contribution to the International Energy Agency (IEA) program on Research and Development in Heat Transfer and Heat Exchangers. 


\title{
Application of Chaos Theory in Identification of Two-Phase Flow Patterns and Transitions in a Small, Horizontal, Rectangular Channel
}

\author{
Y. Cai, M. W. Wambsganss, and J. A. Jendrzejczyk
}

\begin{abstract}
Various measurement tools of chaos theory were applied to analyze twophase pressure signals with the objective to identify and interpret flow pattern transitions for two-phase flows in a small, horizontal rectangular channel. These measurement tools included power spectral density function, autocorrelation function, pseudo-phase-plane trajectory, Lyapunov exponents, and fractal dimensions. It was demonstrated that the randomlike pressure fluctuations characteristic of two-phase flow in small rectangular channels are chaotic in nature. As such, they are governed by a high-order deterministic system. The correlation dimension is potentially a new approach for identification of certain two-phase flow patterns and transitions.
\end{abstract}

\section{Introduction}

This study presents an application of chaos theory in identification of twophase flow patterns and transitions in a small, horizontal, rectangular channel. The data analyzed in this study is from previous experiments (Wambsganss et al., 1991; Wambsganss et al., 1992a and 1992b). In the experiments, horizontal two-phase flow was studied in a small cross-sectional-area $(19.05 \times 3.18 \mathrm{~mm})$ rectangular channel. Adiabatic flows of air/water mixtures were tested over a large mass flux 
range (50 - $\left.2000 \mathrm{~kg} / \mathrm{m}^{2} \mathrm{~s}\right)$. The full quality covered the range experimentally achievable. The two-phase flow patterns and transitions had been identified by dynamic pressure measurements, together with visual observations and supplemented with photographic data, and flow pattern maps were developed (Wambsganss et al., 1991; Wambsganss et al., 1992a and 1992b).

Usually, the flow pattern maps of two-phase flows are based on visual identification of phase distribution (Clarke and Blundell 1989; Brauner and Maron, 1992; Galbiati and Andreini, 1992; Koizumi, 1992 and Hibiki et al., 1992). While visual flow pattern identification may be adequate for some cases, for many situations these methods are inapplicable or too subjective. Several other methods have been developed to more objectively identify and interpret flow pattern and transitions of two phase flow, such as pressure/time signals (Weisman et al., 1979), RMS of pressure/time series and friction pressure gradients (Wambsganss et al., 1991; Wambsganss et al., 1992a and 1992b), the power spectral density function (PSD), probability density function (PDF) (Hubbard and Dukler, 1966; Matsui, 1984 and 1986; Tutu, 1982 and 1984; Vince and Lahey, 1982). These studies have all contributed to understanding of flow patterns and transitions of two-phase flows, but there is no accepted method to objectively distinguish flow patterns.

The purpose of this study is to apply the chaos theory on experimental data of dynamic pressure-to-time signals of two-phase flows in an attempt to identify and interpret flow pattern transitions. This new approach may present a promising way in identification of flow patterns.

Chaotic oscillations are the emergence of randomlike motions from completely deterministic systems. Such motions had been known in fluid mechanics, but they have only recently been observed in low-order mechanical and electrical systems. The recognition that chaotic vibrations can arise in low-order, nonlinear deterministic systems raises the hope of understanding the source of 
randomlike noise and being able to do something about it. The new discoveries in nonlinear dynamics bring with them new concepts and tools for detecting chaotic vibrations in physical systems and for quantifying this "deterministic noise" with new measures such as fractal dimensions and Lyapunov exponents (Moon, 1987).

Two-phase flows and their nonlinear dynamics are far more complicated than other physical systems. Only a few reports with application of chaos theory can be found in literature (Lahey, 1991). Some theoretical developments and "computer experiments" in chaotic dynamics of multi-phase flows have been investigated recently (Dorning 1989a and 1989b; Lahey et al., 1989; Rizwan-uddin and Dorning, 1986; Rizwan-uddin et al., 1988; Rizwan-uddin, 1989). Some experimental investigations of two-phase (gas-solid) flow were conducted, and while the two-phase flow was believed to be deterministic chaos, it could not be characterized with a low-dimensional strange attractor (Tam and Davine, 1989 and 1992). Asymptotic power spectrum analysis and fractal dimension estimation have been employed for characterizing chaotic behavior of two-phase flows in fluidized beds (Ding and Tam, 1993). The power spectral density function, probability density function, and correlation dimension calculations are used by Franca et al. (1991) in an attempt to identify two-phase flow regimes in a $19 \mathrm{~mm}$ i.d. Plexiglas pipe.

In this study, time history data of dynamic pressure $\mathrm{p}_{1}$ from previous experiments (Wambsganss et al., 1991; Wambsganss et al., 1992 a and 1992b) were processed with various measuring tools, such as power spectral density (PSD), autocorrelation function, pseudo-phase-plane trajectory, Lyapunov exponents, and fractal dimensions. It was demonstrated that the randomlike pressure fluctuations characteristic of two-phase flow in small rectangular channels are chaotic in nature. As such, they are governed by a high-order deterministic 
system. The correlation dimension is potentially a new approach for identification of certain two-phase flow patterns and transitions.

\section{Experiment}

Experiments of two-phase flow in a small, horizontal, rectangular channel were conducted several years ago (Wambsganss 1990, 1991, 1992a and 1992b).

The flow apparatus (illustrated schematically in Fig. 1) is designed to allow adiabatic flow experiments with air/liquid mixtures in channels of small crosssectional area. Air is supplied from a compressed air storage tank and flows through a pressure regulator and preselected rotameter to an air/liquid mixer. Laboratory water was used as the liquid in the experiments. The water flows through a control valve and preselected rotameter to the mixer, where air is injected into the liquid stream through a porous medium in opposing walls of the flow channel. The two-phase mixture then flows through the transparent channel. The mixture exiting from the channel flows through an expansion to a drain. A vane-type dry gas meter was utilized to calibrate the gas rotameters, and a weighing-technique-with-stop-watch was used to calibrate the liquid rotameters. The estimated uncertainty in flow rate measurement is $\pm 3 \%$. The flow channel is rectangular, $1.14 \mathrm{~m}$ in length, with cross-sectional dimensions of $19.05 \times 3.18 \mathrm{~mm}$ (aspect ratio of 6). (The aspect ratio is defined as the ratio of the height of the vertical side of the channel to the width of the horizontal side.)

The measured dependent variable is pressure. Pressure taps are spaced at intervals of $114 \mathrm{~mm}$ along the entire length of the channel and are located at the center of the long (vertical) side. Both differential pressure, over a specified channel length, and dynamic pressures, at two locations, are measured. Differential pressure is measured with a strain-gauge-type transducer (Viatran Model 209), and dynamic pressure is measured with piezoresistive-type 
transducers (Endevco Model 8510B-50). The pressure transducers were calibrated against a known standard. Relative to the exit of the mixer, the pressure taps used with the dynamic-pressure-measuring transducers, $\mathrm{p}_{1}$ and $\mathrm{p}_{2}$, are located at $L / \mathrm{D}_{\mathrm{h}}$ equal to 79 and 142, respectively. The channel length over which the differential pressure measurement is made corresponds to an $L / D_{h}$ ratio of 132 . The estimated uncertainty in pressure measurements is $\pm 5 \%$.

The test procedure consisted of establishing total mass flux $G$ and a mass quality $\mathrm{x}$ in the test section. As overall test channel pressure drop allowed, test were performed over a range of quality (typically $10^{-4}$ to 1 ) for each mass flux used (50 to $2000 \mathrm{~kg} / \mathrm{m}^{2} \mathrm{~s}$ ). At steady state, visual and photographic observations were made and pressures were measured. Multiple photographs were taken and pressure measurements were recorded and averaged on a computer-controlled data acquisition system.

From experimental results, typical pressure/time history, RMS pressure and frictional pressure gradient data in the form of two-phase frictional multipliers plotted as a function of both mass quality $\mathrm{x}$ and Martinelli parameter $\mathrm{X}$, together with visual observations and supplemented with photographic data, were summarized in previous work (Wambsganss 1990, 1991, 1992a and 1992b). Such results were used to identify two-phase flow patterns and transitions and to develop flow pattern maps for two-phase flow in small, horizontal, rectangular channel.

\section{Chaotical Measurements}

In this study, a group of measured data of dynamic pressure $p_{1}$ (see Fig. 1) in flow regimes of plug, slug and annular flows, with a fixed mass flux $G=500$ $\mathrm{kg} / \mathrm{m}^{2} \mathrm{~s}$, were investigated by various chaotical measurement tools. Typical pressure/time histories, exhibiting different characteristics corresponding to the different flow patterns (plug, slug and annular flows), are shown in Fig. 2. It is 
demonstrated that different flow patterns of two-phase flows in the small channel always exhibit a randomlike pressure fluctuation characteristics, even though plug and slug flow are characterized by low-frequency and annular flow is characterized by high-frequency.

Various measurement tools of chaos theory were applied in this study to analyze dynamic pressure signals of two-phase flows with the objective to identify and interpret flow pattern transitions. These measurement tools included power spectral density function, autocorrelation function, pseudo-phase-plane trajectory, Lyapunov exponents, and fractal dimensions.

The experimental pressure data were obtained from original recorded tapes at sampling rate of 1000 digitizations per second with Macintosh machine. The data length of 65,000 points for each test case were utilized for calculations of various chaotic measurements, especially, for algorithm of computing the fractal dimension.

\subsection{Power Spectral Density}

Power spectral density (PSD) makes it possible to distinguish between periodic and chaotic responses. It is known that for a chaotic motion, the power spectrum is a continuous process.

The PSD analysis can give a first indication of the dimensional behavior of a time series. The PSD analysis suggested by Gorman and Robbins (1992) can reveal information complementary to a conventional dimensional analysis or an estimation of Lyapunov exponents. Therefore, this approach should be considered before performing an expensive and time consuming dimensional analysis, like the correlation integral or the "nearest neighbor" approaches.

The characteristics of the power spectrum of chaotic-dynamic systems in the low- to moderate-frequency regimes are reflected as a broad-band structure without 
dominant peak structures. However, at the high-frequency limit of the asymptotic regimes, power spectra decay toward a noise level. That noise level may be determined from instrumentation limit for the data originating from measurements. The manner in which the spectrum decays toward the noise level contains useful information on the underlying dynamics. Some researches have suggested that the asymptotic analysis of power spectra is very useful in distinguishing between highand low- to-moderate dimensional chaos (Ding and Tam, 1993).

The PSD of plug, slug and annular flows in the small channel are shown in Fig. 3 on semi-log scale. All of flow regimes exhibit a broad band of frequencies, which is a characteristic of chaotic behavior. At the high-frequency range for all of flow regimes (see Fig. 3), the power spectra show a clear power-law falloff by visual inspection. As suggested by Sigeti and Horsthemke (1987), the power-law falloff of the power spectrum should indicate high-dimensional chaos rather than a stochastic process since the data were generated from a deterministic system.

Therefore, above analysis of the PSD for two-phase flows in the small channel indeed indicates the existence of high dimensional chaotic behavior, and, as we will see later the high dimensional attractors from correlation integral estimations. However, it is not possible to distinguish the flow patterns and transitions of two-phase flows from the analysis of power spectrum.

\subsection{Autocorrelation Function}

Autocorrelation function is another signal processing tool used to identify chaotic motions. When a signal is chaotic, information about its past origins is lost. This means that the signal is only correlated with its recent past.

The autocorrelation functions of the plug, slug and annular flows are shown in Fig. 4. The autocorrelation functions for plug, slug and annular flows have a 
peak at origin delay time $\tau=0$, and drop off rapidly with time, reflecting chaotic behavior.

It can be observed from Fig. 4 that the plug, slug and annular flow have different periodic oscillations along the time delay axis with the amplitude values of autocorrelation functions around zero. However, with limited analysis, we can not claim that those differences can be used to identify flow patterns.

The importance and contribution of calculating the autocorrelation functions before we performing other chaotic measurements is not only that the autocorrelation function has provided a useful tool to distinguish chaotic behavior but also it can provide an optimum choice of time delay $\tau$ for other chaotic measurements. To estimate the attractor dimension, it is necessary to construct the phase space. The phase space may be reconstructed by using the time-delay embedding method; while the calculated values of the correlation dimension and Lyapunov exponents from reconstructed phase space are very sensitive to the time delay $\tau$. Our experience has indicated that it is a very useful and practical approach by choosing an optimum time delay value from the calculations of autocorrelation functions. As from Fig. 4, the optimum time delay is estimated to be the smallest value of $\tau$ at which the first minimum in the autocorrelation function occurs, which is also considered to be most independent. This is the time delay used in subsequent chaotic measurements.

\subsection{Pseudo-Phase-Planes}

To obtain fractal dimension of strange attractor, many methods assume that one knows the dimension of the phase space wherein the attractor lies, or one has the ability to measure all the state variable. However, in our experiments, the time history of only one state variable is available, i.e., dynamic pressure $\mathrm{p}_{1}(\mathrm{t})$. Also, the number of degrees of freedom, or minimum number of significant modes 
contributing to the chaotic dynamics of two-phase flows, are not known a priori. In this case, pseudo-phase-space (embedding space) trajectories of the motion, or strange attractor, can be reconstructed using the time-delay embedding method (Packard et al., 1980). Given a scalar time series, one can reconstruct Ddimensional vectors in the following form:

$$
x_{i}=\left[x\left(t_{i}\right), x\left(t_{i}+\tau\right), x\left(t_{i}+2 \tau\right), \ldots, x\left(t_{i}+(D-1) \tau\right)\right]
$$

where $\mathrm{D}$ is the embedding dimension (the minimum dimension of the subspace), and $\tau$ is the time delay. This reconstructed geometrical structure has the same dimensional characteristics, for example, the correlation dimension of the attractor generated from the dynamics underlying the original scalar time series.

Figure 5 presents pseudo-phase-plane plots of the measured dynamic pressure for plug, slug and annular flows, respectively. It is obvious from these three flow patterns that the pseudo-phase-plane plots indeed indicate chaotic behavior. However, the difference of pseudo-phase-plane signatures among the three flow patterns is not sufficiently clear to be used for distinguishing flow patterns as suggested by Franca et al. (1991).

\subsection{Lyapunov Exponents}

Chaos in dynamics implies a sensitivity of the outcome of a dynamical process to changes in initial conditions. If one imagines a set of initial conditions within a sphere or radius $\varepsilon$ in phase space, then chaotic motion trajectories, originating in the sphere, will map the sphere into an ellipsoid whose major axis grows as $d=\varepsilon e^{\lambda t}$, where $\lambda>0$ is know as a Lyapunov exponent. Lyapunov

exponents are the average exponential rates of divergence or convergence of nearby orbits in phase space (Moon, 1987). 
Lyapunov exponents have been shown to be the most useful dynamic diagnostic tool in determining chaotic systems quantitatively.

A number of experimenters in chaotic dynamics have developed algorithms to calculate the Lyapunov exponent. For regular motions $\lambda \leq 0$; but for chaotic motion $\lambda>0$. Thus, the sign of $\lambda$ is a criterion for chaos. Any system containing at least one positive Lyapunov exponent is defined as chaotic.

We used the algorithms proposed by Wolf et al. (1985) to determine the Lyapunov exponents from a time series of absolute pressure for different flow patterns. Figure 6 shows the results of Lyapunov exponents for plug, slug and annular flows. Lyapunov exponents are positive, which shows that the attractor has an expanding direction, constituting convincing evidence for chaotic behavior of plug, slug and annular flows. The result provides further evidence of chaotic behavior in two-phase flows in small channels.

\subsection{Correlation Dimensions}

Another approach for predicting chaotic motion quantitatively is the use of fractal dimensions. A noninteger fractal dimension of the orbit in a phase space implies the existence of a strange attractor. The basic idea is to characterize the "strangeness" of the chaotic attractor. While practical use of the fractal dimensions in measuring and characterizing chaotic motions has yet to be fully established, various definitions have been developed, including the capacity dimension, correlation dimension, and information dimension.

Of the several methods available to estimate the attractor dimensions, we used measurement of the correlation dimension, which has been used successfully by many investigators in other fields (Moon, 1985). In particular, we used Grassberger and Proccacia's method (1983) to calculate the correlation dimensions of measured pressure data of two-phase flow. 
The correlation function is defined as: $C(r)=\lim _{N \rightarrow \infty}\left(1 / N^{2}\right)$ (number of pairs of points on the attractor whose distance is less than $r$ ). Here, $N$ is the number of D-dimensional vectors constructed out of the scalar time series. For some range $r$, called the scaling region, $C(r)$ scales as $r^{d_{c}}$, where $d_{c}$ is the correlation dimension (Grassberger and Proccacia, 1983). A saturation of $d_{c}$ as the embedding dimension $\mathrm{D}$ increases indicates that the time series has a non-random component.

The correlation integral analysis is applied to the experimental data of pressure in two-phase flows. Figure 7 shows the correlation functions $\mathrm{C}(\mathrm{r})$ versus $\log _{2} \mathrm{r}$ for plug, slug and annular flows, respectively, with embedding dimensions increasing from 5 to 30 in intervals 5 . The correlation dimensions at different embedding dimensions are determined by the slopes of the least-squares fitted straight line of the correlation functions. The estimated correlation dimensions versus the embedding dimensions for plug, slug and annular flows are plotted in Fig. 8, which shows that the correlation dimensions tend to saturate at values of $d_{c}$ $\approx 6.6$ as the embedding dimension reaches 15 or greater for the plug flow, at values of $d_{c} \approx 5.4$ as the embedding dimension reaches 20 or greater for the slug flow, and at values of $d_{c} \approx 9.3$ as the embedding dimension reaches 20 or greater for the annular flow. The results from Figs. 7 and 8 provide evidence that the two-phase flow in the small channel are deterministic chaotic motions not a random oscillation (the dimension of random oscillation will increase as the embedding dimension increases).

It appears that the correlation dimension may provide a new tool in identification of two-phase flow patterns and transitions in a small, horizontal, rectangular channel. 


\section{Identifying Flow Patterns and Transitions}

The correlation functions $C(r)$ with various mass quality $x$ in three regimes of plug-bubble, slug and annular flows are shown in Fig. 9 (with embedding dimension $d_{E}=20$ ). For the plug-bubble flows, the correlation functions fall in the region of $\log _{2} r=-5$ to 0 , and with mass quality $x$ increasing, the correlation function advances to right (see Fig. 9 (a)); for the slug flows, the correlation functions fall in the region of $\log _{2} \mathrm{r}=-1$ to 3 , and with mass quality $\mathrm{x}$ increasing, the correlation function again shifts to the right (see Fig. 9 (b)); for the annular flows, the correlation functions fall in the region of $\log _{2} \mathrm{r}=1$ to 3 , and with mass quality $\mathrm{x}$ increasing, the correlation function shifts to the left (see Fig. 9 (c)). Figure 9 shows a tendency of the correlation function to distinguish flow patterns for two-phase flows in small channels.

Correlation dimensions as a function of mass quality $\mathrm{x}$ are compared with RMS pressures in Fig. 10. We can identify a difference in fractal dimension levels for the various flow regimes, even though all would be defined as highdimensional chaos. When plotted as a function of mass quality, a change in trend of the correlation dimension could be identified with the plug/bubble-to-slug flow pattern transition. With increasing mass quality, the correlation dimension decreases in the plug/bubble region and increases in the slug region. the correlation dimension was also lower in the plug/bubble region. The transition to annular flow could not be determined from the correlation dimension data. For their plug/bubble-to-slug flow transition the result is in good agreement with the result of RMS of dynamic pressure-time signals (Wambsganss et al., 1990, 1991, 1992a and 1992b).

From Figs. 9 and 10, we can see there apparently exist changes in the trend of the fractal dimension estimation that can be used to distinguish flow patterns and 
transitions, as these two-phase flows in small channels are, in fact, deterministic systems.

\section{Conclusions}

Various measurement tools of chaos theory were applied to analyze twophase pressure signals with the objective to identify and interpret flow pattern transitions. These measurement tools included power spectral density function, autocorrelation function, pseudo-phase-plane trajectory, Lyapunov exponents, and fractal dimensions.

Application of the pseudo-phase-plane trajectory, the power spectral density (PSD) and autocorrelation functions indeed indicated the existence of chaotic behavior in the two-phase pressure measurement. In particular, the pseudo-phaseplane trajectory displayed complicated patterns, the PSD was continuous, and the autocorrelation function showed a peak at $\tau=0$ and then dropped off rapidly. The Lyapunov exponents were shown to be greater than zero in quality ranges corresponding to all the various flow patterns of interest. This provides the most convincing evidence of chaotic behavior and distinguishes the two-phase flows in the small, horizontal, rectangular channel as a deterministic chaos rather than a random system. However, the results from these various measurement tools of chaotic motion, such as the PSD function, autocorrelation function, pseudo-phaseplane trajectory, Lyapunov exponents, can not be easily adopted as criteria for flow pattern transition identification.

The theory of fractal dimensions, in the form of correlation dimensions, was used to quantify the identified chaotic behavior. Embedding techniques and approach to obtain the optimum time delay were employed to calculate the correlation dimensions. In general, high dimensions were found for two-phase pressure signals in ranges corresponding to all the various flow patterns of interest. 
When plotted as a function of mass quality, a change in trend of correlation dimension could be identified with the plug/bubble-to-slug flow pattern transition. The correlation dimension decreased as the mass quality increased in the plug/bubble region, while the correlation dimension increased as the mass quality increased in the slug region. The correlation dimension was lower in the plug/bubble region.

It appears that fractal dimensions offer a promising way to objectively classify flow patterns. However, the time for data processing is such that it is not a practical tool for on-line analysis. The software we used for dimension estimations are not well developed. Algorithms should be improved so that more data points can be involved and processing time can be reduced. Then, not only the accuracy of chaotic measurements will be increase greatly, but also the method of fractal dimension estimations can be easily applied in broad practical purposes.

It was demonstrated that the randomlike pressure fluctuations, characteristic of two-phase flow in small rectangular channels, are chaotic in nature. As such, they are governed by a high-order deterministic system. The correlation dimension is potentially a new approach for identification of certain two-phase flow patterns and transitions. However, more work will be required before objective techniques are available for identification and classification of two-phase flow patterns and transitions. We hope this study will stimulate the performance of future research relating to the application of chaos theory in this important area.

\section{Acknowledgments}

This work was supported by the U.S. Department of Energy, Office of Conservation and Renewable Energy, Division of Advanced Industrial Concepts, and represents a U.S. contribution to the International Energy Agency (IEA) program on Research and Development in Heat Transfer and Heat Exchangers. 


\section{References}

Brauner, N. and Maron D. M., 1992, "Identification of the range of 'small diameters' conduits, regarding two-phase flow pattern transitions," Int. Comm. Heat Mass Transfer, Vol. 19. pp. 29-39.

Clarke, r. H. and Blundell, N., 1989, "The two-phase flow patterns occurring inside a plane-fin passage of a plate-fin heat exchanger," Proceedings of AIChE Symposium Series, Heat Transfer - Philadelphia, vol. 85, pp. 287-292.

Ding, J. and Tam, S. W., 1993, "Asymptotic power spectrum analysis of chaotic behavior in fluidized beds," Int. J. of Bifurcation and Chaos (to be published).

Dorning, J., 1989, "Nonlinear dynamics and chaos in heat transfer and fluid flow," Proceedings of AIChE Symposium Series, Heat Transfer - Philadelphia, vol. 85, pp. 13-29.

Dorning, J., 1989, "An introduction to chaotic dynamics in two-phase flow," Proceedings of AIChE Symposium Series, Heat Transfer - Philadelphia, vol. 85, pp. 241-248.

Franca, F., Acikgoz, M., Lahey Jr., R. T. and Clausse, A., 1991, "The use of techniques for flow regime identification," Int. J. Multiphase Flow, Vol. 17, No. 4. pp. 545-552.

Galbiati, L. and Andreini, P., 1992, "Flow pattern transition for vertical downward two-phase flow in capillary tubes. inlet mixing effects," Int. Comm. Heat Mass Transfer, Vol. 19. pp. 791-799.

Gorman, M. and Robbins, K. A., 1992, "Real-time identification of Flame Dynamics," in Applied Chaos, ed. Kim, J. H. and Stringer, J., John Wiley \& Sons, New York, pp. 261-276. 
Grassberger, P. and Procaccia, I., 1983, "Measuring the Strangeness of Strange Attractors," Physica 9D, pp. 189-208.

Hibiki, T., Mishima, K., Yoneda, K., Fujine, S., Kanda, K. and Nishihara, H., 1992, "Visualization and measurement of gas-liquid two-phase flow in a narrow rectangular duct with use of the neutron radiography and image processing technique," ANS Proceedings, 1992 National Heat Transfer Conference, Aug. 9-12, 1992, San Diego, CA., Vol. 6. pp. 101-108.

Hubbard, M. G. and Dukler, A. E., 1966, "The characterization of flow regimes for horizontal two-phase flow," Proc. Heat Transfer and Fluid Mechanics Institute. Stanford Univ. Press, Stanford, Calif.

Koizumi, Y, 1992, "Air/water two-phase flow in a horizontal large-diameter pipe -flow regime and pressure drop--," ANS Proceedings, 1992 National Heat Transfer Conference, Aug. 9-12, 1992, San Diego, CA., Vol. 6. pp. 309-320.

Lahey, R. T. , 1991, "An Application of Fractal and Chaos Theory in the Field of Two-Phase Flow and Heat Transfer," Warmw- und Stoffubertragung 26, 351363 .

Lahey, R. T., Clause, A. and DiMarco, P., 1989, "Chaos and non-linear dynamics of density-wave instabilities in a boiling channel," Proceedings of AIChE Symposium Series, Heat Transfer - Philadelphia, vol. 85, pp. 256-261.

Matsui, G., 1984, "Identification of flow regimes in vertical gas liquid two-phase flow using differential pressure fluctuations," Int. J. Multiphase Flow, 10, pp. 711-720.

Matsui, G., 1986, "Automatic identification of flow regimes in vertical two-phase flow using differential pressure fluctuations," Nucl. Engng. Des. 95, pp. 221231.

Moon, F. C., 1987, Chaotic Vibration, New York: John Wiley. 
Packard, N., Muench, J., Schwartz, C., Mees, A. I. and Rapp, P. E., 1980, "Geometry from a time series," Phys. Rev. Lett., 45, 712.

Press, W. H., Flannery, B. P., Teukolsky, S. A., and Vetterling, W. T., 1986, Numerical Recipes, Cambridge University Press, Cambridge.

Rizwan-uddin and Dorning, J. J., 1986, "Some nonlinear dynamics of a heated channel," Nuclear Science and Engineering: 93, 1-14.

Rizwan-uddin and Dorning, J. J., 1988, "A chaotic attractor in a periodically forced two-phase flow system," Nuclear Science and Engineering: 100, 393-404.

Rizwan-uddin, 1989, "Strange attractors in forced two-phase flow heated channels," Proceedings of AIChE Symposium Series, Heat Transfer Philadelphia, vol. 85, pp. 249-255.

Sigeti, D. and Horsthemke, W., 1987, "High-frequency power spectrum for systems subject to noise," Phys. Rev. A 35(5), pp. 2276-2282.

Tam, S. W. and Davine, M., 1989, "Is There a Strange Attractor in a Fluidized Bed?" in Measures of Complexity and Chaos, ed. Abraham, N. B. et al., Plenum Press, New York, pp. 193-197.

Tam, S. W. and Davine, M., 1992, "A Study of Fluidized-Bed Dynamical Behavior: A Chaos Perspective," Applied Chaos, ed. Kim, J. H. and Stringer, J., John Wiley \& Sons, New York, pp. 381-393.

Tutu, N. K., 1982, "Pressure fluctuations and flow pattern recognition in vertical two-phase gas-liquid flow," Int. J. Multiphase Flow, 8, pp. 443-447.

Tutu, N. K., 1984, "Pressure drop fluctuations and bubble-slug transition in a vertical two-phase water flow," Int. J. Multiphase Flow, 10, pp. 211-216.

Vince, M. A. and Lahey, R. T., 1982, "On the development of an objective flow regime indicator, "Int. J. Multiphase Flow 8, pp. 93-124. 
Wambsganss, M. W., Jendrzejczyk, J. A., and France, D. M., 1991, "Two-Phase Flow Patterns and Transitions in a Small, Horizontal, Rectangular Channel", Int. J. Multiphase Flow Vol. 17, No. 3, pp. 327-342.

Wambsganss, M. W., Jendrzejczyk, J. A., and France, D. M., 1992, "Determination and Characteristics of the Transition to Two-Phase Slug Flow in a Small Horizontal Channel", ASME, HTD-Vol. 220, Two-Phase Flow in Energy Exchange Systems, pp. 63-71, to be published in ASME, J. Fluid Engineering.

Wambsganss, M. W., Jendrzejczyk, J. A., France, D. M., and Obot, N. T., 1992, "Frictional Pressure Gradients in Two-Phase Flow in a Small Horizontal Rectangular Channel", Experimental Thermal and Fluid Science; 5:40-56.

Weisman, J., Duncan, O., Gibson, J., and Crawford, T., 1979, "Effects of Fluid Properties and Pipe Diameter in Two-phase Flow Patterns in Horizontal lines, " Int. J. Multiphase Flow, 5, 437-462.

Wolf, A., Swift, J. B., Swinney, H. L., and Vastano, J. A., 1985, "Determining Lyapunov Exponents from a Time Series," Physica 16D, 285-317. 


\section{Figure Captions}

Fig. 1 Schematic illustration of adiabatic two-phase flow apparatus

Fig. 2 Time histories of dynamic pressure $p_{1}(t)$ for (a) plug, (b) slug and (c) annular flows

Fig. 3 PSDs of dynamic pressure $\mathrm{p}_{1}(\mathrm{t})$ for (a) plug, (b) slug and (c) annular flows

Fig. 4 Autocorrelation functions of dynamic pressure $p_{1}(t)$ for (a) plug, (b) slug and (c) annular flows

Fig. 5 Pseudo-phase planes of dynamic pressure $p_{1}(t)$ for (a) plug, (b) slug and (c) annular flows

Fig. 6 Lyapunov exponents of dynamic pressure $\mathrm{p}_{1}(\mathrm{t})$ for (a) plug, (b) slug and (c) annular flows

Fig. 7 Correlation dimensions of dynamic pressure $\mathrm{p}_{1}(\mathrm{t})$ for (a) plug, (b) slug and (c) annular flows

Fig. 8 Correlation dimensions $d_{C}$ vs. embedding dimension $d_{E}$ for plug, slug and annular flows

Fig. 9 Correlation dimensions of dynamic pressure $\mathrm{p}_{1}(\mathrm{t})$ for (a) plug, (b) slug and (c) annular flows with embedding dimension $\mathrm{d}_{\mathrm{E}}=20$

Fig. 10 Comparison of correlation dimension and RMS of pressure $\mathrm{p}_{1}(\mathrm{t}) \mathrm{vs.}$ mass quality $x$ 


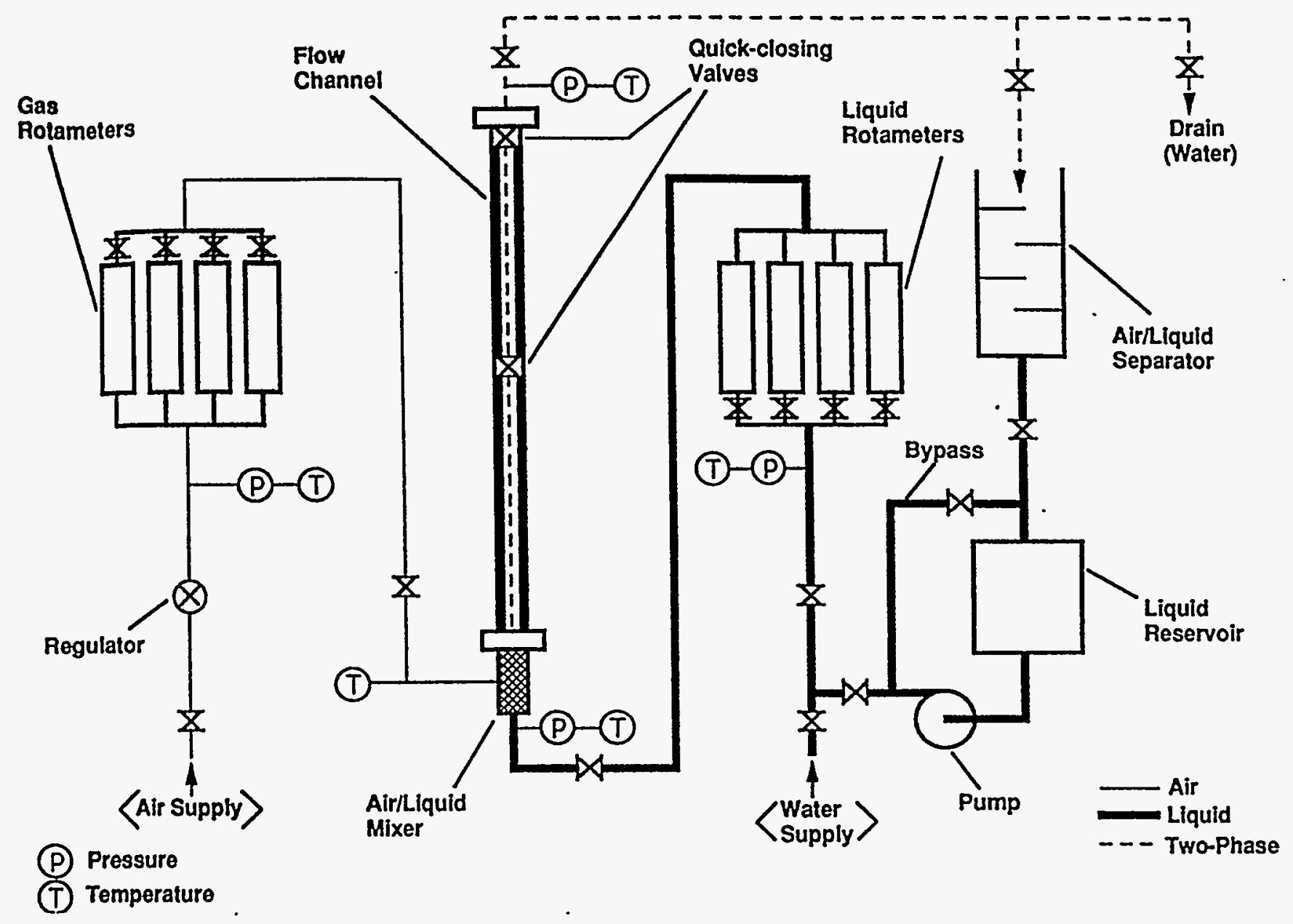

Fig. 

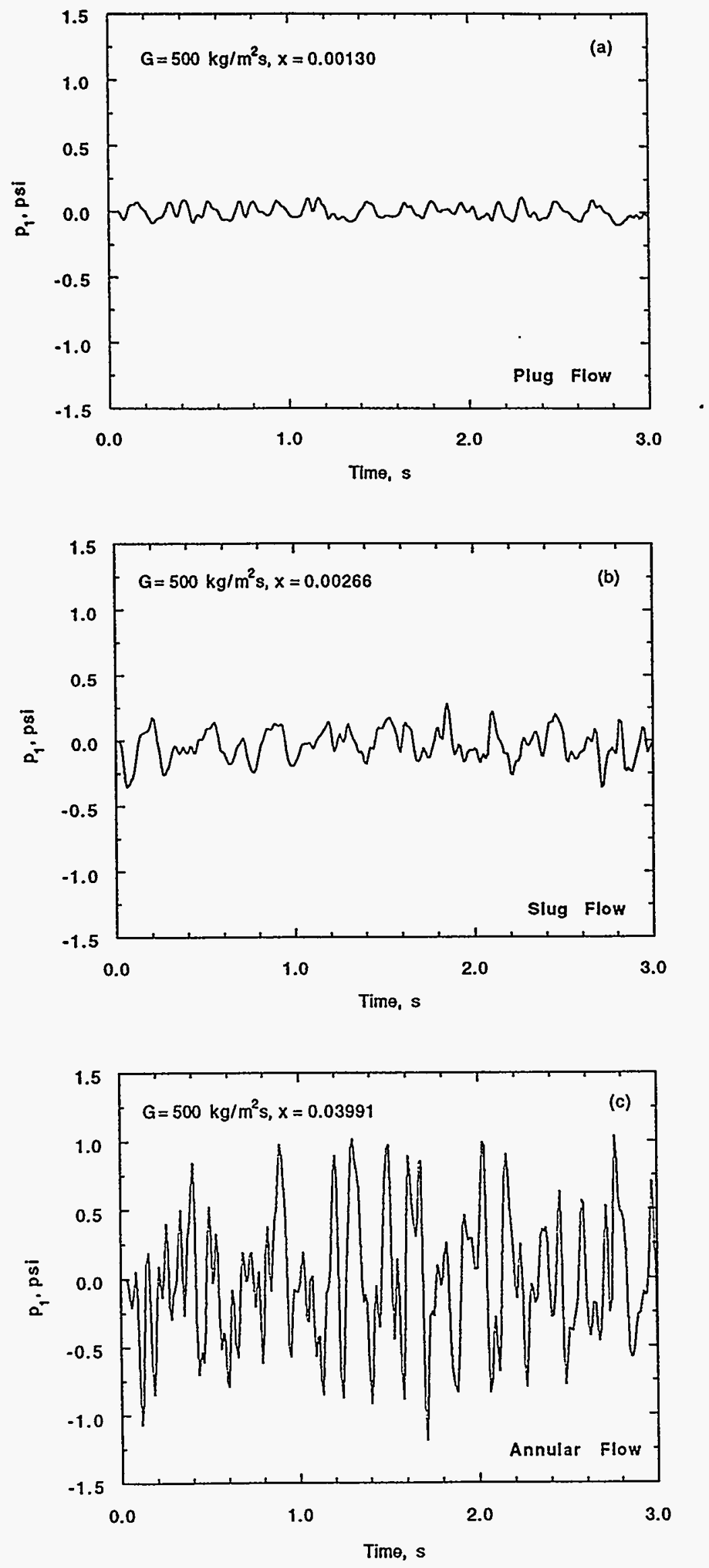

$F i r 2$ 

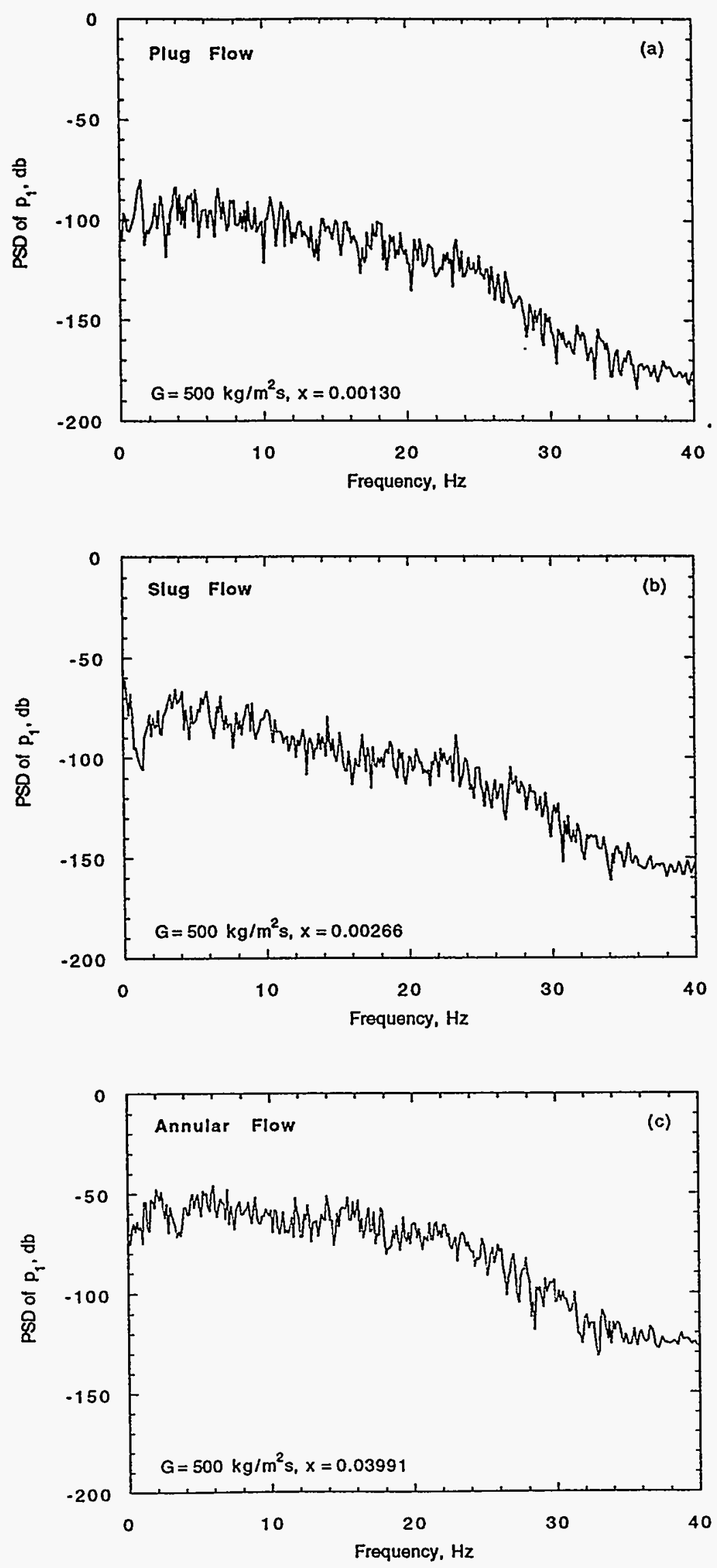

Fig 3 

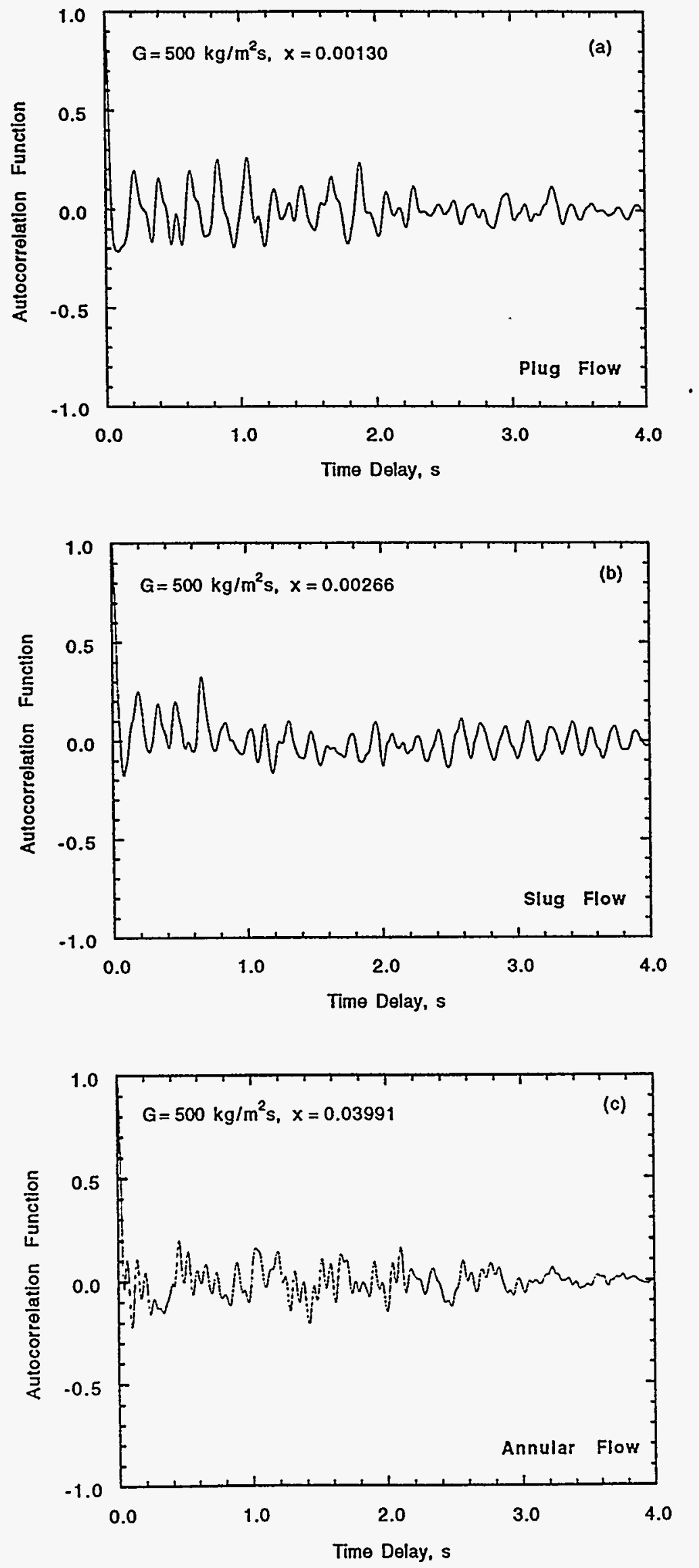

$F: 64$ 

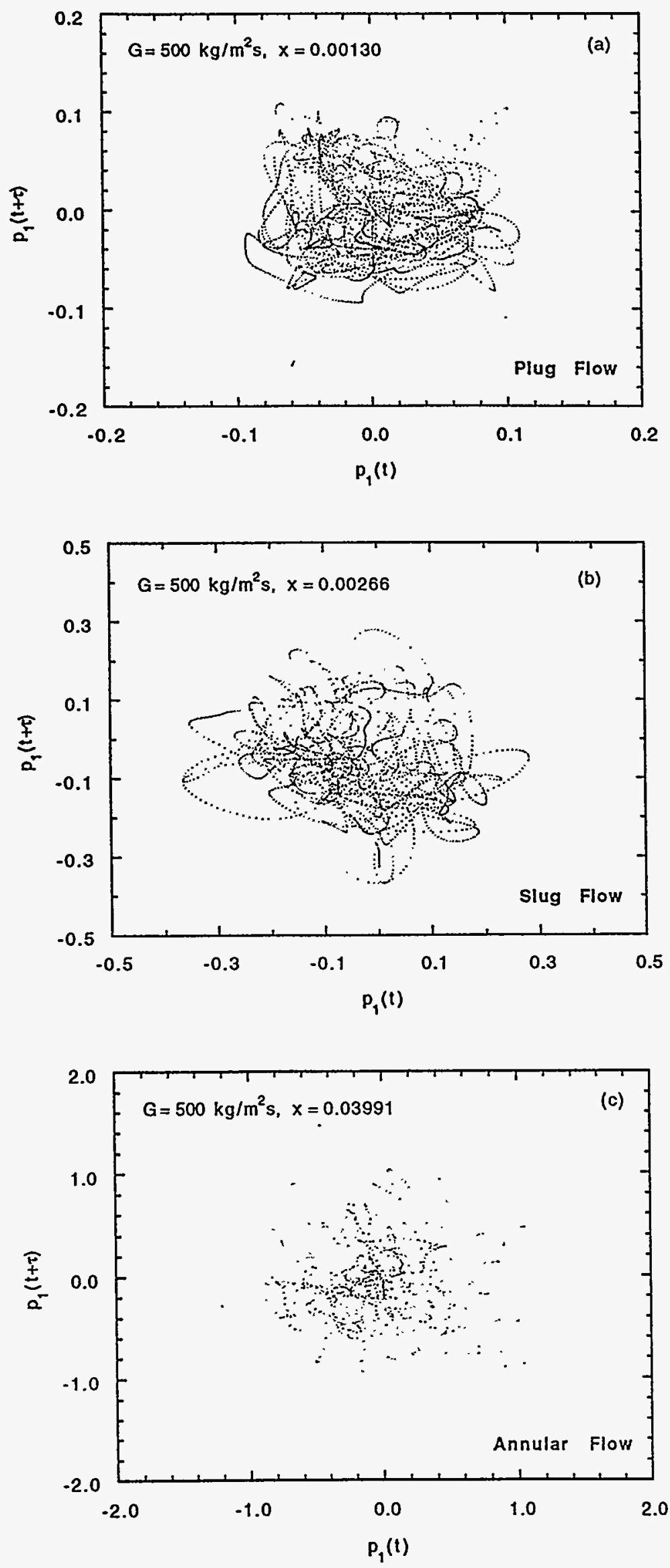

Fig. 5 

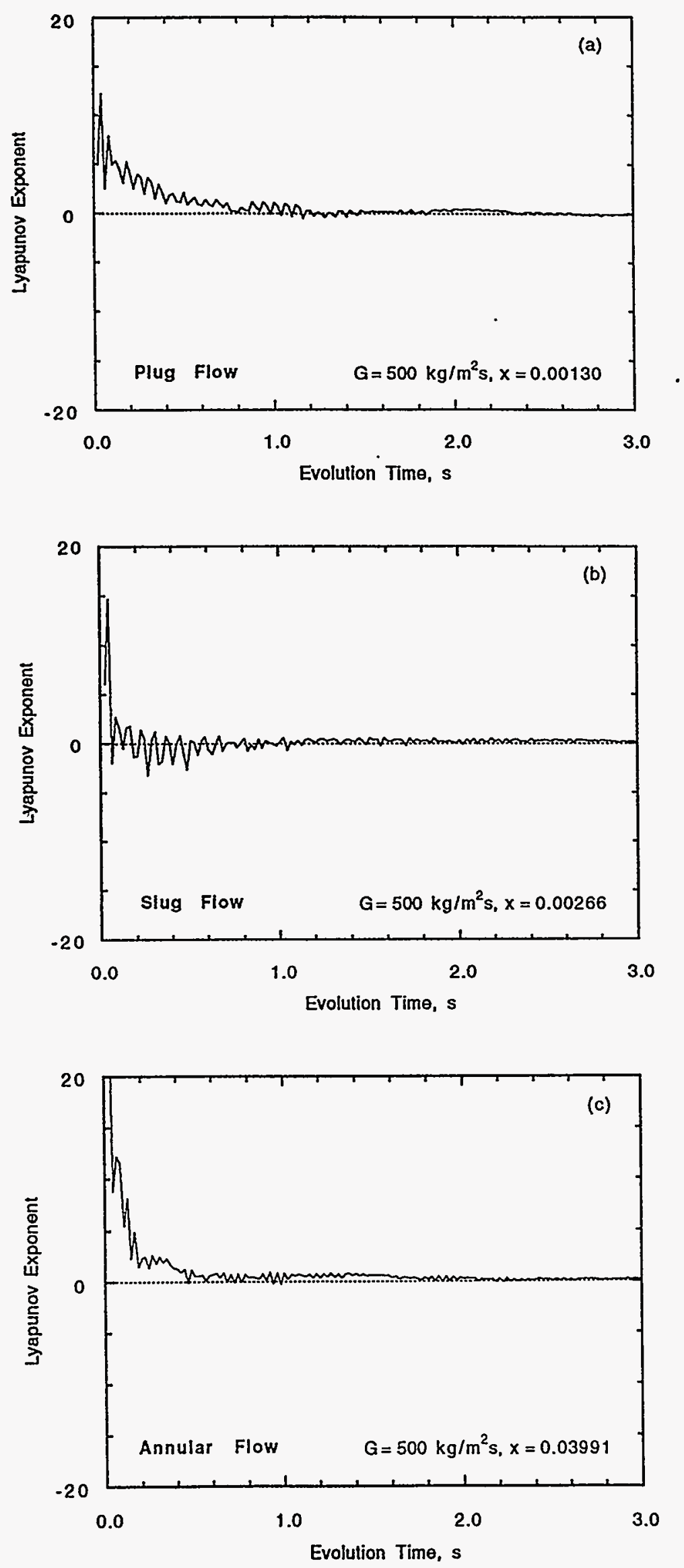

Fig. 6 

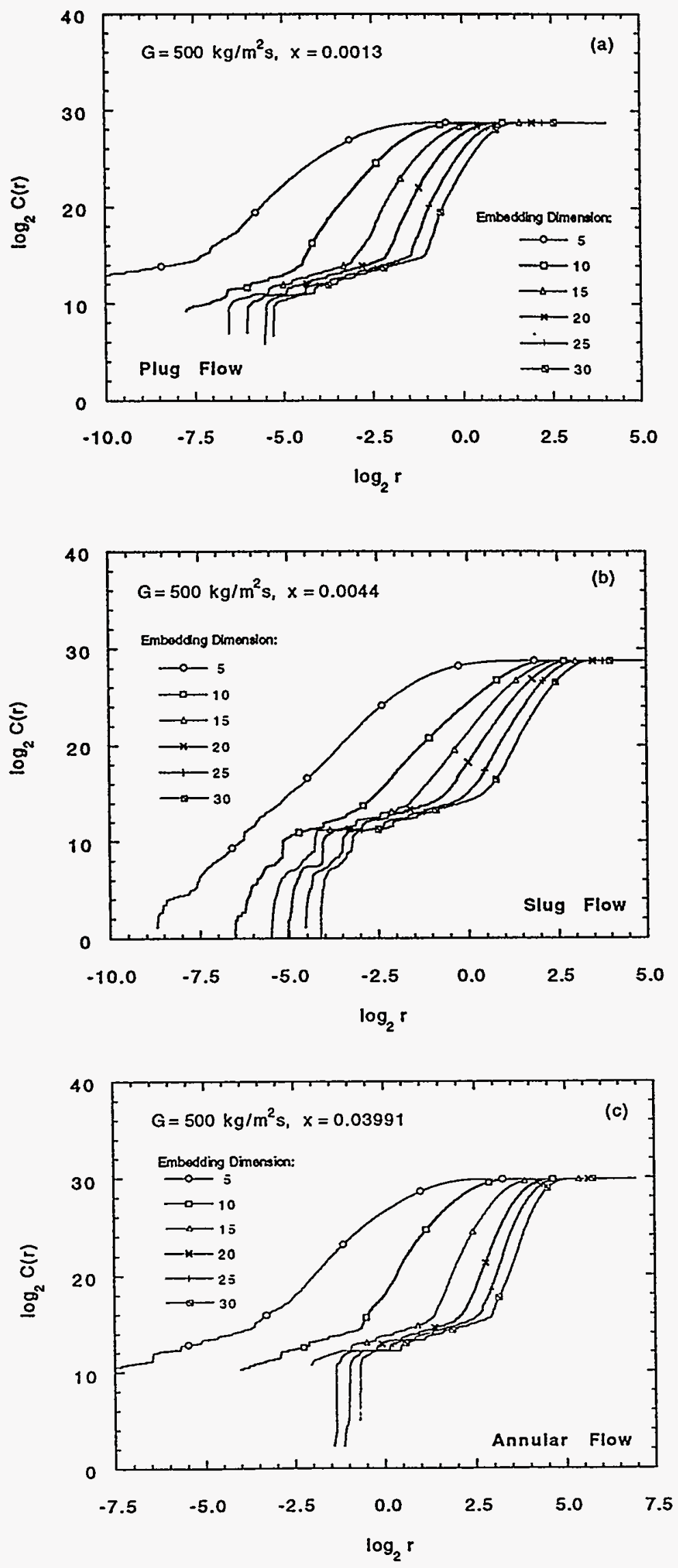


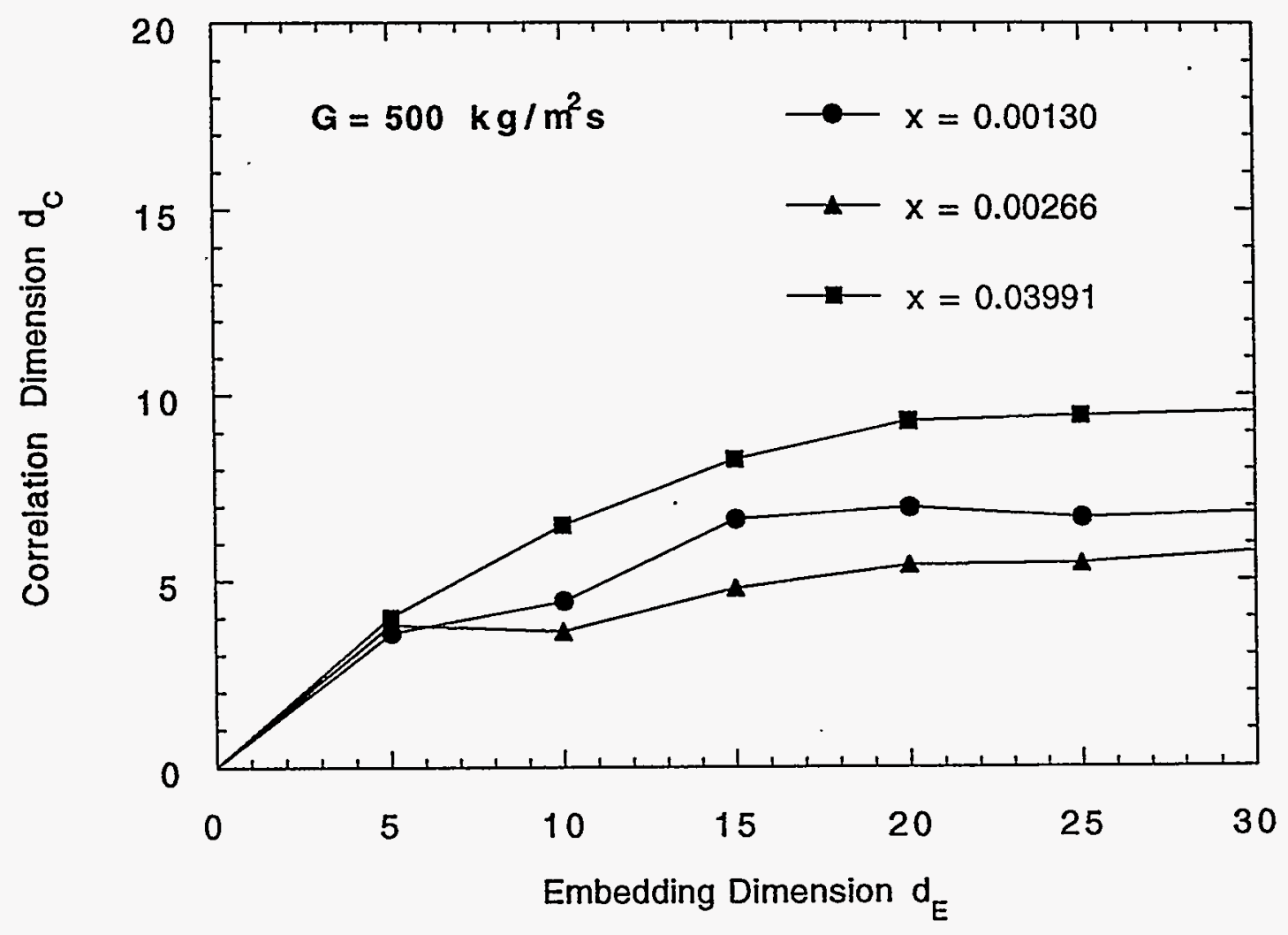

$\therefore$ : 

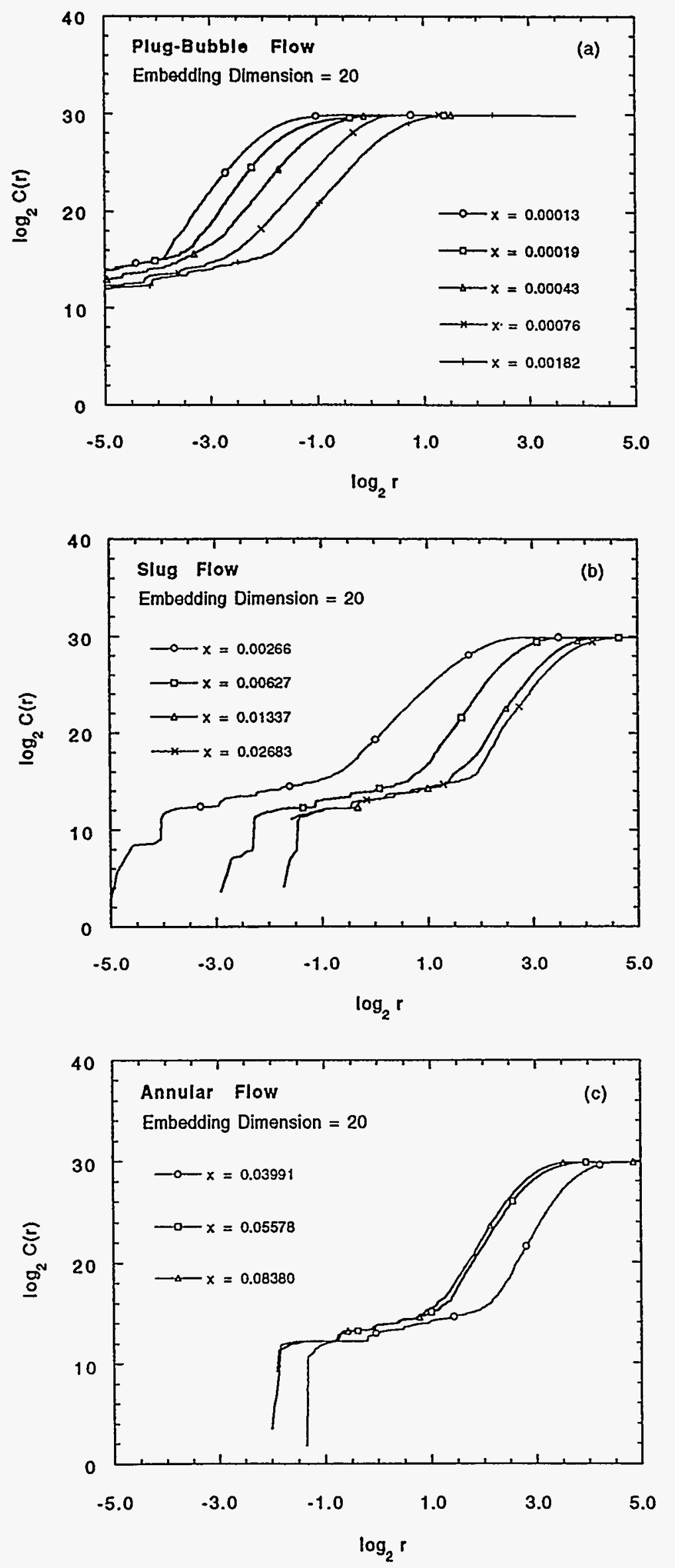

Fig 9 


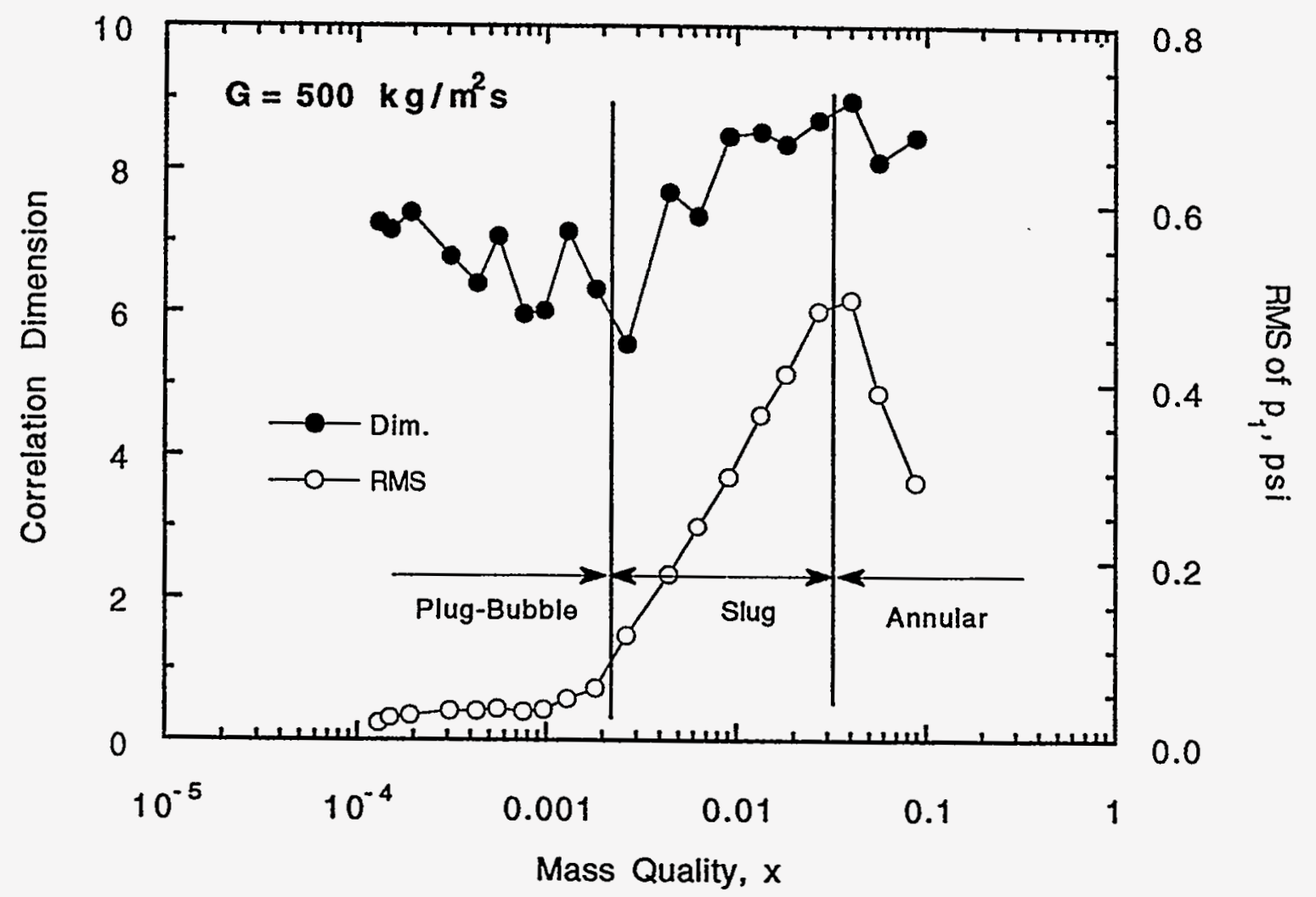

\section{DISCLAIMER}

This report was prepared as an account of work sponsored by an agency of the United States Government. Neither the United States Government nor any agency thereof, nor any of their employees, makes any warranty, express or implied, or assumes any legal liability or responsibility for the accuracy, completeness, or usefulness of any information, apparatus, product, or process disclosed, or represents that its use would not infringe privately owned rights. Reference herein to any specific commercial product, process, or service by trade name, trademark, manufacturer, or otherwise does not necessarily constitute or imply its endorsement, recommendation, or favoring by the United States Government or any agency thereof. The views and opinions of authors expressed herein do not necessarily state or reflect those of the United States Government or any agency thereof.

Fig. 10 